\title{
Modified embedded atom method study of the mechanical properties of carbon nanotube reinforced nickel composites
}

\author{
Jamal Uddin, ${ }^{1,2, *}$ M. I. Baskes, ${ }^{3}$ S. G. Srinivasan, ${ }^{2,4}$ Thomas R. Cundari, ${ }^{1,2}$ and Angela K. Wilson ${ }^{1,2}$ \\ ${ }^{1}$ Department of Chemistry, University of North Texas, 1155 Union Circle \#305070, Denton, Texas 76203, USA \\ ${ }^{2}$ Center for Advanced Scientific Computing \& Modeling, University of North Texas, Denton, Texas 76203, USA \\ ${ }^{3}$ Materials Science and Technology Division, Los Alamos National Laboratory, MS G755, Los Alamos, New Mexico 87545, USA \\ ${ }^{4}$ Department of Materials Science \& Engineering, University of North Texas, Denton, Texas 76203, USA
}

(Received 12 October 2009; published 11 March 2010)

\begin{abstract}
We report an atomistic simulation study of the behavior of nanocomposite materials that are formed by incorporating single-walled carbon nanotubes (SWCNTs), with three different diameters, and a multiwalled carbon nanotube (MWCNT) into a single-crystal nickel matrix. The interactions between carbon and nickel atoms are described by a modified embedded atom method potential. Mechanical properties of these nanocomposite materials are predicted by atomistic calculations and compared with that of fcc nickel and pristine CNTs. Our simulations predict that all Ni/CNT composites studied in this work are mechanically stable. Their elastic properties depend on the volume fraction and diameter of embedded CNTs. The single-crystal Young's modulus $\left(E_{11}\right)$ of Ni/SWCNT composites exhibit a large increase in the direction of CNTs alignment compared to that of a single-crystal nickel. However, a moderate but gradual decrease is seen for $E_{22}$ and $E_{33}$ in the transverse directions with increase in CNT diameters. As a consequence, Ni/SWCNTs show a gradual decrease for the polycrystalline Young's, bulk and shear moduli with the increasing CNT diameters and volume fractions. These reductions, although moderate, suggest that enhancement of mechanical properties for polycrystalline Ni/SWCNT nanocomposites are not achievable at any CNT volume fraction. The Ni/MWCNT composite with high CNT volume fraction shows the highest increase in $E_{11}$. Unlike the $E_{22}$ and $E_{33}$ for $\mathrm{Ni} /$ SWCNTs, there is a significant increase in the $E_{22}$ and the $E_{33}$ for Ni/MWCNT. As a result, polycrystalline Ni/MWCNT composites show slight increase in the elastic properties. This suggests that nickel nanocomposites with enhanced mechanical properties can be fabricated using large volume fractions of larger diameter MWCNTs. Depending on type, alignment and volume fraction, Ni/CNT composites show varying degrees of elastic anisotropy and Poisson's ratio compared to pure Ni. Simulation predicts strong adhesion at the Ni/CNT interface and a significant interfacial stress transfer between CNT and Ni matrix.
\end{abstract}

DOI: 10.1103/PhysRevB.81.104103

PACS number(s): 62.23.Pq, 81.40.Jj, 68.35.Gy, 62.20.de

\section{INTRODUCTION}

Carbon nanotubes (CNTs) are among the stiffest and strongest materials. ${ }^{1}$ Their extremely small size, high aspect ratios, and extraordinary structural and chemical stability make CNTs a leading candidate for reinforcement fillers in nanocomposite materials. ${ }^{2-6}$ As a result, there has been an increasing scientific and technological interest in developing nanocomposites by dispersing CNTs into a metal matrix or by laser depositing metal matrix around the CNTs. Such composites combine low density with high strength and stiffness, and enhanced electrical and thermal properties. These are particularly attractive for aerospace and other transportation applications ${ }^{2-4}$ where there is a renewed emphasis on increasing fuel efficiency by "light weighting" the transporters.

CNT-metallic composites offer distinct advantages over polymeric composites for high-strength applications. ${ }^{5,6} \mathrm{Ku}-$ zumaki et al. were the first to study a aluminum/CNT composite. ${ }^{7}$ Preparation and application of magnesium/CNT composites was also reported. ${ }^{8,9}$ The mechanical strength of nickel/CNTs nanocomposites was studied recently by Sun $e t$ al. ${ }^{10}$ Some progress has also been made in the application of atomistic simulations to predict the elastic and the mechanical properties of such composite materials. For example, molecular-dynamics (MD) simulations have been success- fully applied to predict the elastic properties of polyethylene/ CNT composites ${ }^{11}$ and other polymeric CNT composites. ${ }^{12}$ However, despite their potential superiority in mechanical properties, the development of CNT-metal matrix composites remains in its infancy. Development of metal matrix composites will be promoted from atomistic insights on the response of their mechanical properties to chemical and material modifications.

Nickel is the primary ingredient of numerous aerospace superalloys, ${ }^{13,14}$ and therefore, is an attractive target for fabrication of ultrastrength materials with CNTs. This research investigates the mechanical properties of pristine CNTs, a fcc nickel matrix, and Ni/CNT composites at different CNT volume concentrations using quasistatic atomistic simulations. Elastic properties of solids are important because they relate to various fundamental solid-state properties. They determine the response of the crystal to external forces, as characterized by elastic constants, bulk modulus, shear modulus, Young's modulus, and Poisson's ratio. Therefore, elastic properties play an important part in determining the strength of the materials. We have calculated these properties and the elastic anisotropy of $\mathrm{Ni} / \mathrm{CNT}$ nanocomposite materials. Interatomic interactions are described using a modified embedded atom method (MEAM) initially developed by Daw and Baskes ${ }^{15,16}$ and later modified by Baskes. ${ }^{17}$ 
TABLE I. Parameters for the MEAM potentials of Ni, C, and Ni-C. The parameters are the cohesive energy $E_{c}(\mathrm{eV})$, the equilibrium nearest-neighbor distance $r_{e}(\AA)$, the exponential decay factor for the universal energy function $\alpha$, the scaling factor for the embedding energy A, the four exponential decay factors for the atomic densities $\beta^{(i)}$, the four weighting factors for the atomic densities $t^{(\mathrm{i})}$, and the density scaling factor $\rho^{0}$. Angular screening parameters for the MEAM potentials for binary systems.

\begin{tabular}{|c|c|c|c|c|c|c|}
\hline \multicolumn{4}{|c|}{ MEAM potentials } & \multicolumn{3}{|c|}{ Angular screening parameters } \\
\hline & $\mathrm{Ni}$ & $\mathrm{C}$ & $\mathrm{Ni}-\mathrm{C}$ & & $C_{\max }$ & $C_{\min }$ \\
\hline$E_{c}$ & 4.45 & 7.37 & 4.82 & $\mathrm{Ni}-\mathrm{Ni}-\mathrm{Ni}$ & 2.8 & 0.8 \\
\hline$r_{e}$ & 2.49 & 1.54 & 2.01 & $\mathrm{Ni}-\mathrm{C}-\mathrm{Ni}$ & 2.8 & 2.0 \\
\hline$\alpha$ & 4.99 & 4.38 & 4.82 & $\mathrm{C}-\mathrm{Ni}-\mathrm{C}$ & 2.8 & 2.0 \\
\hline A & 1.10 & 1.49 & & $\mathrm{Ni}-\mathrm{Ni}-\mathrm{C}$ & 2.8 & 2.0 \\
\hline$\beta^{(0)}$ & 2.45 & 4.26 & & $\mathrm{Ni}-\mathrm{C}-\mathrm{C}$ & 2.8 & 2.0 \\
\hline$\beta^{(1)}$ & 1.50 & 5.00 & & $\mathrm{C}-\mathrm{C}-\mathrm{C}$ & 2.8 & 2.0 \\
\hline$\beta^{(2)}$ & 6.00 & 3.20 & & & & \\
\hline$\beta^{(3)}$ & 1.50 & 3.98 & & & & \\
\hline$t^{(0)}$ & 1.00 & 1.00 & & & & \\
\hline$t^{(1)}$ & 5.79 & 7.50 & & & & \\
\hline$t^{(2)}$ & 1.60 & 1.04 & & & & \\
\hline$t^{(3)}$ & 3.70 & -1.01 & & & & \\
\hline$\rho^{0}$ & 1.00 & 1.00 & & & & \\
\hline
\end{tabular}

\section{COMPUTATIONAL DETAILS}

\section{A. Theoretical formalism}

In MEAM, the total energy of a system is given in the following form:

$$
E=\sum_{i}\left[F_{i}\left(\bar{\rho}_{i}\right)+\frac{1}{2} \sum_{j(\neq i)} S_{i j} \varphi_{i j}\left(R_{i j}\right)\right],
$$

where $F_{i}$ is the embedding function for an atom $i$ embedded in a background electron density $\bar{\rho}_{i}, S_{i j}$ is the screening function, and $\varphi_{i j}\left(R_{i j}\right)$ is the pair interaction between atoms $i$ and $j$ separated by a distance $R_{i j}$. For the calculations of energy, the functional forms of $F_{i}$ and $\varphi_{i j}$ should be given. The background electron density at each atomic site is computed by combining several partial electron-density terms for various angular contributions with weight factors $t^{(h)}(h=0-3)$ (directionality of bonding). Each partial electron density is a function of atomic configuration and atomic electron density. The atomic electron densities $\rho^{a(h)}(h=0-3)$ are given as

$$
\rho^{a(h)}(R)=\rho_{0} \exp \left[-\beta^{(h)}\left(R / r_{e}-1\right)\right],
$$

where $\rho_{0}$, the atomic electron-density scaling factor, and $\beta^{(h)}$, the decay lengths, are adjustable parameters, and $r_{e}$ is the nearest-neighbor (NN) distance in the equilibrium reference structure. A specific form is given to the embedding function $F_{i}$ but not to the pair interaction $\varphi_{i j}$. Instead, a reference structure where individual atoms occupy perfect-crystal atom sites is defined and the potential energy per atom of the reference structure is estimated from the zero-temperature universal equation of state by Rose et al. ${ }^{18}$ The value of the pair interaction is evaluated from the known values of the potential energy per atom and the embedding energy, which is a function of the nearest-neighbor distance.
In the original MEAM, ${ }^{17}$ only the first nearest-neighbor interactions were considered. The second ( $2 \mathrm{NN})$ and more distant neighbor interactions were neglected by using strong many-body screening function. The second-nearest-neighbor interactions have been included in the modified formalism by adjusting screening parameters $C_{\min }$ to decrease the manybody screening effect. In addition, a radial cutoff function is also applied to reduce calculation time. The MEAM for an alloy system is based on the MEAM potentials for the component elements. The 2 NN MEAM formalism ${ }^{19}$ gives 14 independent model parameters for pure elements: four $\left(E_{c}, r_{\mathrm{e}}, B, d\right)$ for the universal equation of state, seven $\left(\beta^{(0)}, \beta^{(1)}, \beta^{(2)}, \beta^{(3)}, t^{(1)}, t^{(2)}, t^{(3)}\right)$ for the electron density, one $(A)$ for the embedding function, and two $\left(C_{\min }, C_{\max }\right)$ for the many-body screening. The details of the MEAM formalism have been published in the literature. ${ }^{17,20,21}$

\section{B. Simulation methods}

The MEAM potential parameters that describe the interatomic interactions for $\mathrm{Ni}, \mathrm{C}$, and $\mathrm{Ni}-\mathrm{C}$ composites are listed in the Table I. It treats nickel, carbon, and the $\mathrm{Ni}-\mathrm{C}$ interactions, and allows the formation of chemical bonds with appropriate atomic hybridization. Similar MEAM potential parameters have been shown to model the catalytic growth of CNTs on nickel nanocatalysts well. ${ }^{22}$ We performed molecular static calculations at $T=0 \mathrm{~K}$. Periodic boundary conditions were applied along all three directions of the supercells. The Ni matrices and $\mathrm{Ni} / \mathrm{CNT}$ composites were relaxed to minimize their energies.

The Ni/CNT composites were simulated using three single-walled nanotubes (SWCNTs) with varying diameters and a multiwalled (MWCNT) nanotube. We chose CNTs with zigzag structures for this study. SWCNTs with $(5,0)$, 


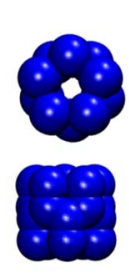

SWCNT(5,0)

(a)

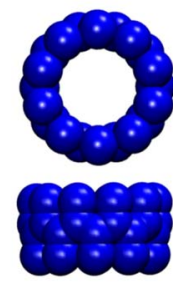

SWCNT(10,0)

(b)

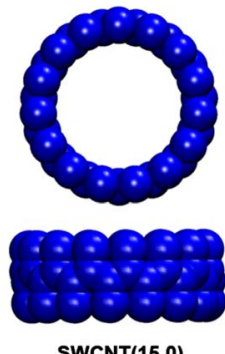

(c)

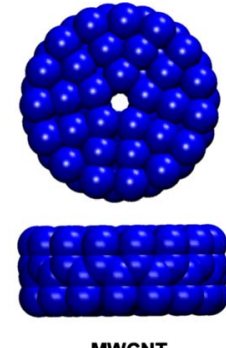

(d)
FIG. 1. (Color online) Space filling unit cells of $(n, 0)$ CNTs. SWCNT and MWCNT refer to single-walled and multiwalled CNT. (a) $\operatorname{SWCNT}(5,0)$, (b) $\operatorname{SWCNT}(10,0)$, and (c) $\operatorname{SWCNT}(15,0)$. MWCNT in (d) is built by assembling $\operatorname{SWCNT}(5,0)$, SWCNT $(10,0)$, and SWCNT $(15,0)$ nanotubes coaxially. The approximate tubular height for the unit cell is $4.26 \AA$ for all nanotubes.

$(10,0)$, and $(15,0)$ chiral indices and tubular diameters of $3.97 \AA$, $7.86 \AA$, and $11.77 \AA$, respectively, were studied. These CNTs were embedded in a supercell containing 37044 nickel atoms. The atomic structure and the crystallographic orientation of the SWCNTs and supercells are shown in Figs. 1 and 2 . This supercell has $21 \times 21 \times 21$ unit cells and is approximately $7.4 \mathrm{~nm} \times 7.4 \mathrm{~nm} \times 7.4 \mathrm{~nm}$ in three perpendicular directions.

As the CNT volume fraction is an important variable in determining the composite mechanical properties, we used two different concentrations of each CNTs for Ni/CNT composites. The three supercells had a CNT volume fraction of $0.83 \%, 1.85 \%$, and $3.37 \%$ when single CNTs were used. When four CNTs are embedded in each supercell, the CNT

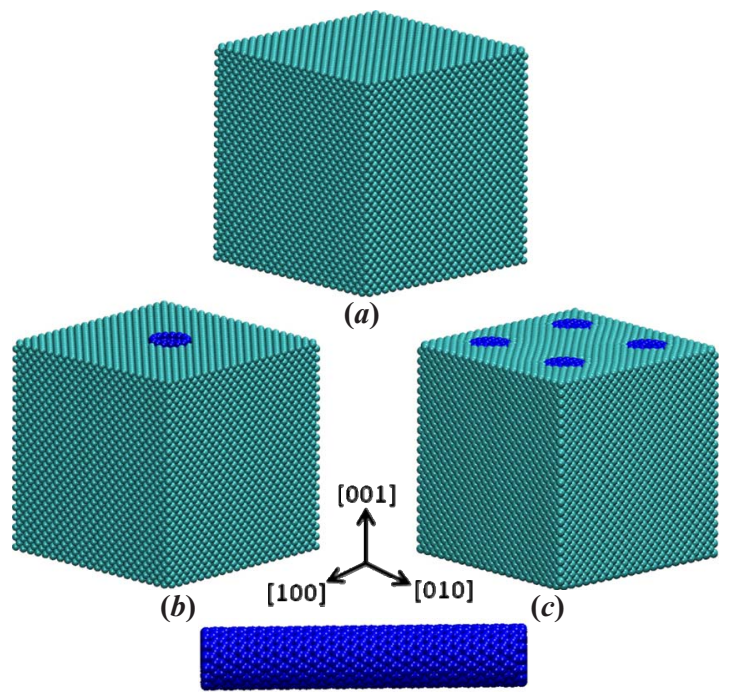

(d)

FIG. 2. (Color online) Atomistic structures and the crystallographic orientations of the supercells. (a) Fully minimized pure nickel (face-centered-cubic lattice), (b) nickel matrix with an embedded MWCNT, (c) nickel matrix with four embedded MWCNT, (d) full extent of a MWCNT embedded in the nickel supercell. Periodic boundary conditions are applied for pure nickel and $\mathrm{Ni} /$ CNT matrix along the three dimensions. volume fractions were increased to $3.33 \%, 7.40 \%$, and $13.46 \%$ for $(5,0),(10,0)$, and $(15,0)$ SWCNTs, respectively. Since the Ni matrix does not penetrate the hollow inside of CNT, we treated the CNTs as solid beams for volume fractions calculations.

The MWCNTs were built by inserting SWCNTs into each other coaxially while maintaining their individual periodicity. For composites made with MWCNTs, the CNT volume fractions were similar to the volume fractions of SWCNT(15,0). For example, Fig. 2(d) shows MWCNT that fits the periodicity of fcc Ni matrix. The wall thickness of SWCNTs were assumed to be $0.32 \mathrm{~nm}$ based on electrondensity distribution calculations ${ }^{23}$ and similar methods. ${ }^{24,25}$

To simulate the Ni/CNTs nanocomposites with low CNT volume fractions, four different single-crystal $\mathrm{Ni}$ supercells containing a hollow cylindrical void at the center were built. For studying nanocomposites with high CNT volume fractions, four different single-crystal $\mathrm{Ni}$ supercells were built with four cylindrical voids approximately equidistant to each other. The cylindrical void runs from one face to the opposite face of the supercell with the cylinder axis aligned parallel to the supercell [001] direction ( $a$ axis). The radii of these voids were chosen to fit $\operatorname{SWCNT}(5,0), \quad \operatorname{SWCNT}(10,0)$, SWCNT $(15,0)$, and the MWCNT, leaving an appropriate amount of space for Ni-C interatomic bonding. The nanocomposites were formed by embedding the CNTs into the voided Ni supercells. The resulting composites contain a homogeneous distribution of aligned CNTs. Two simulation cells with embedded CNTs are shown in Figs. 2(b) and 2(c).

Mechanical properties and stress-strain relationships were determined from the relaxed configurations. Elastic constants for $\mathrm{Ni}$ and $\mathrm{Ni} / \mathrm{CNTs}$ were calculated from strain-energyapplied strain relationships by fitting the strain energy per volume to a parabola. Strains of $0.1 \%$ deformation around the fully minimized structures were chosen to maintain deformations within the linear elastic regime. For each deformation step (0.0002 step size), we minimized system energy by relaxing positions of all atoms. Nine-independent elastic constants $\left(c_{11}, c_{22}, c_{33}, c_{12}, c_{23}, c_{31}, c_{44}, c_{55}\right.$, and $\left.c_{66}\right)$ and three independent constants $\left(c_{11}, c_{12}\right.$, and $\left.c_{44}\right)$ of Ni/CNTs and pure fcc Ni were determined in this study. The directiondependent Young's modulus $(E),{ }^{26}$ which measures the stiffness of a material, was calculated from the slope of the strain-stress curves for pristine CNTs. Young's modulus for CNTs with only longitudinal response were calculated with 0.0002 increments in the applied strain. For $\mathrm{Ni}$ matrix and $\mathrm{Ni} / \mathrm{CNT}$ systems, $E$ was calculated from the appropriate single-crystal elastic constants.

\section{RESULTS AND DISCUSSION}

\section{A. Pristine CNTs}

Table II shows that the Young's moduli of individual CNTs obtained in our work agree well with numerous published experimental ${ }^{28,30,31,33}$ and theoretical values. ${ }^{22,24,27,43}$ Most of the experimental measurements of $E$ were carried out for MWCNTs. Also, Young's moduli of SWCNTs show a large variation from 0.78 to $3.6 \mathrm{TPa}$ and that only a few experimental results are available. ${ }^{33,35,37,44,45}$ Our calculated 
TABLE II. Young's modulus $\left(E_{11}\right)$ (in GPa) for single-walled (SWCNTs) and a multiwalled (MWCNT) carbon nanotubes obtained from our MEAM Ni-C potential are tabulated and compared with the literature values.

\begin{tabular}{|c|c|c|}
\hline This work & Previous calculations & Experiments \\
\hline & $820(\text { Xiao et al., MD })^{\mathrm{a}}$ & \\
\hline & 900 (Agrawal et al., MD) $^{\mathrm{b}}$ & 270-900 (Yu et al. $)^{\mathrm{c}}$ \\
\hline & $5500\left(\right.$ Yakobson, MD) ${ }^{\mathrm{d}}$ & $790(\mathrm{Li} \text { et } \text { al. })^{\mathrm{e}}$ \\
\hline $552[\operatorname{SWCNT}(5,0)]$ & $974(\mathrm{Lu}, \mathrm{MD})^{\mathrm{f}}$ & $900\left(^{(D e m c y z e k} \text { et al. }\right)^{\mathrm{g}}$ \\
\hline $832[\operatorname{SWCNT}(10,0)]$ & $1325(\text { Cheng and Gao, MD })^{\mathrm{h}}$ & $816 \pm 410(\text { Salvetat } \text { et } \text { al. })^{\mathrm{i}}$ \\
\hline $892[\operatorname{SWCNT}(15,0)]$ & 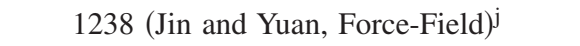 & $1200(\text { Tombler et al. })^{\mathrm{k}}$ \\
\hline \multirow[t]{8}{*}{740 (MWCNT) } & 1220 (Hernández et al., TB-MD) ${ }^{1}$ & $1250(\text { Krishnan et al. })^{\mathrm{m}}$ \\
\hline & $4700(\mathrm{Tu}, \mathrm{LDA})^{\mathrm{n}}$ & \\
\hline & $764{\text { (Zhou et al., LCAO-MO cluster) }{ }^{\circ}}^{\circ}$ & \\
\hline & $965,972(\text { Ogata and Shibutani, TB, DFT })^{\mathrm{p}}$ & \\
\hline & $1470($ Pullen, GGA)q & \\
\hline & $1060(\mathrm{Li} \text { and Chou, MM) })^{\mathrm{r}}$ & \\
\hline & $899(5,0), 916(10,0), 923(15,0)($ Meo and & \\
\hline & Rossi, MM-FEM) $)^{\mathrm{s}}$ & \\
\hline
\end{tabular}

$\begin{array}{ll}{ }^{\mathrm{a} R e f e r e n c e ~} 22 . & { }^{\mathrm{f}} \text { Reference } 24 . \\ { }^{\mathrm{b}} \text { Reference } 27 . & \text { gReference } 31 . \\ { }^{\mathrm{c}} \text { Reference } 28 . & \text { hReference } 32 . \\ { }^{\mathrm{d}} \text { Reference } 29 . & { }^{i} \text { Reference } 33 . \\ { }^{\mathrm{e}} \text { Reference } 30 . & { }^{j} \text { Reference } 34 .\end{array}$

$E$ of $832 \mathrm{GPa}$ for $\operatorname{SWCNT}(10,0)$ agrees with the value calculated by Xiao et al. (820 GPa), Agrawal et al. ${ }^{27}$ (900 GPa), and $\mathrm{Lu}(975 \mathrm{GPa}) .{ }^{24}$ Our results from the same CNT is also in reasonable agreement with the values of 965 and $972 \mathrm{GPa}$ calculated using tight-binding and density-functional theory, respectively, by Ogata and Shibutani. ${ }^{40}$ On the contrary, Hernández and coworkers ${ }^{36}$ reported a much greater value of $1220 \mathrm{GPa}$ for this nanotube using tight-binding MD. These large differences may be attributed to differences in the CNT volume estimation due to variations in effective CNT wall thickness. In spite of extensive studies, the variations in wall thickness of CNTs remain an unresolved question. ${ }^{46}$

\section{B. Ni/CNT composites:}

\section{Single-crystal elastic constants}

A necessary condition for mechanical stability of a structure is that its strain energy must be positive against arbitrary but small homogeneous elastic deformations. For an orthorhombic crystal, this imposes the following constraints: ${ }^{47}$ $c_{11}+c_{22}>2 c_{12}, \quad c_{22}+c_{33}>2 c_{23}, \quad c_{11}+c_{33}>2 c_{31}, \quad c_{i i}>0 \quad(i$ $=1-6)$, and $c_{11}+c_{22}+c_{33}+2 c_{12}+2 c_{23}+2 c_{31}>0$. These criteria simplify to $c_{11}>0, c_{44}>0 \quad c_{11}+2 c_{12}>0$, and $c_{11}-c_{12}$ $>0$ for cubic crystals such as fcc Ni. Hence, we examined the mechanical stability of the Ni/CNT composites using appropriate single-crystal elastic constants for composites with both low and high CNT volume fractions. The calculated values for elastic constants $c_{i j}$ are summarized in Table III.

For single-crystal $\mathrm{Ni}$, the MEAM calculated values for $c_{11}, c_{12}$, and $c_{44}$ agree well with experiments. ${ }^{48,49}$ The shear elastic constants $c_{11}-c_{12}$ and $c_{11}+2 c_{12}$ determine stability.

$\begin{array}{ll}{ }^{\mathrm{k}} \text { Reference } 35 . & \\ { }^{\mathrm{l}} \text { Reference } 36 . & { }^{\mathrm{p}} \text { Reference } 40 . \\ { }^{\mathrm{m}} \text { Reference } 37 . & { }^{\mathrm{T}} \text { Reference } 41 . \\ { }^{\mathrm{n}} \text { Reference } 38 . & { }^{\mathrm{s}} \text { Reference } 42 .\end{array}$

${ }^{\circ}$ Reference 39.

Physically, $c_{44}$ plays a subtle role and represents the resistance of the system to a shear deformation such as in the (100) plane in the [010] direction. Similarly, $\left(c_{11}-c_{12}\right) / 2$ represents the resistance to shear deformation across, for example, a (110) plane in the [1 $\overline{1} 0]$ direction. Similar observations hold for an orthorhombic crystal. From the Table III data, it is observed that for Ni/CNT composites, the $c_{i i}(i$ $=1-6), \quad c_{11}+c_{22}-2 c_{12}, \quad c_{22}+c_{33}-2 c_{23}, \quad c_{11}+c_{33}-2 c_{13}$, and $c_{11}+c_{22}+c_{33}+2 c_{12}+2 c_{23}+2 c_{13}$ are all positive, and thus it can be reasonably concluded that Ni/CNTs composites are mechanically stable. This is an encouraging result for the fabrication of nanocomposite materials by embedding the CNTs in metal matrices.

For Ni/CNTs, $c_{11}$ show moderate increase in composites with low CNT volume fractions and significantly large increase in composites with high CNT volume fractions compared to that of pure $\mathrm{Ni}$. These increases depend on the type and diameter size of the embedded nanotubes. For example, composites Ni/SWCNT(5,0), Ni/SWCNT(10,0), and $\mathrm{Ni} /$ SWCNT $(15,0)$ show an increase of 5,10 , and $12 \mathrm{GPa}$, respectively, for low CNT volume fraction and 19, 39, and 42 $\mathrm{GPa}$, respectively, for high $\mathrm{CNT}$ volume fraction. $\mathrm{Ni} /$ MWCNT shows the largest increase in $c_{11}$ than for any of the $\mathrm{Ni} / \mathrm{SWCNTs}$. In this case, $c_{11}$ shows an increase of $30 \mathrm{GPa}$ and $109 \mathrm{GPa}$, respectively, for low and high CNT volume fractions compared to that of $\mathrm{Ni}$. In the transverse directions, $c_{22}$ or $c_{33}$ show gradual decrease for Ni/SWCNTs compared to pure Ni. However, in these cases, composites with higher CNT volume fractions show a larger decrease compared to composites with low CNT volume fractions. Interestingly, the Ni/MWCNT nanocomposite shows very small or no changes for $c_{22}$ or $c_{33}$. 
TABLE III. Single-crystal elastic constants $c_{i j}$ (in GPa), and derived quantities $c_{11}+c_{22}-2 c_{12}, c_{22}+c_{33}-2 c_{23}, c_{11}+c_{33}-2 c_{13}$, and $c_{11}$ $+c_{22}+c_{33}+2 c_{12}+2 c_{23}+2 c_{13}$ for pure fcc nickel and the Ni/SWCNTs and Ni/MWCNT composites with low and high CNT volume fractions.

\begin{tabular}{|c|c|c|c|c|c|c|c|c|c|}
\hline & \multirow[t]{2}{*}{$\mathrm{Ni}(\mathrm{fcc})$} & \multicolumn{2}{|c|}{ Ni/SWCNT $(5,0)$} & \multicolumn{2}{|c|}{ Ni/SWCNT(10,0) } & \multicolumn{2}{|c|}{ Ni/SWCNT(15,0) } & \multicolumn{2}{|c|}{ Ni/MWCNT } \\
\hline & & Low & High & Low & High & Low & High & Low & High \\
\hline$c_{11}$ & 247.1 & 251.9 & 266.1 & 256.7 & 284.0 & 258.8 & 289.4 & 276.9 & 356.1 \\
\hline$c_{22}$ & 247.1 & 246.0 & 243.6 & 245.4 & 241.5 & 243.2 & 233.4 & 245.7 & 243.9 \\
\hline$c_{33}$ & 247.1 & 246.0 & 243.9 & 245.2 & 240.6 & 243.4 & 233.6 & 246.3 & 247.5 \\
\hline$c_{12}$ & 147.5 & 146.6 & 144.0 & 145.5 & 139.7 & 142.7 & 129.7 & 142.9 & 132.8 \\
\hline$c_{23}$ & 147.5 & 148.3 & 150.5 & 147.9 & 148.8 & 147.7 & 147.5 & 149.1 & 149.6 \\
\hline$c_{13}$ & 147.5 & 148.0 & 144.9 & 145.5 & 140.1 & 142.7 & 129.5 & 143.6 & 133.8 \\
\hline$c_{44}$ & 125.4 & 123.7 & 118.6 & 118.5 & 99.6 & 114.5 & 83.8 & 118.2 & 109.5 \\
\hline$c_{55}$ & 125.4 & 121.4 & 112.3 & 121.9 & 113.1 & 120.7 & 109.1 & 118.4 & 104.7 \\
\hline$c_{66}$ & 125.4 & 121.4 & 112.3 & 121.9 & 113.1 & 120.7 & 109.1 & 118.4 & 104.8 \\
\hline$c_{11}+c_{22}-2 c_{12}$ & 199.3 & 204.7 & 221.7 & 211.1 & 246.1 & 216.6 & 263.8 & 236.8 & 334.3 \\
\hline$c_{22}+c_{33}-2 c_{23}$ & 199.3 & 201.3 & 220.1 & 210.9 & 244.4 & 216.8 & 263.6 & 236.1 & 294.9 \\
\hline$c_{11}+c_{33}-2 c_{13}$ & 199.3 & 195.5 & 186.5 & 194.8 & 184.5 & 191.2 & 172.0 & 193.8 & 184.5 \\
\hline$c_{11}+c_{22}+c_{33}+2 c_{12}+2 c_{23}+2 c_{13}$ & 1626.2 & 1630.3 & 1632.5 & 1625.1 & 1623.3 & 1611.6 & 1569.8 & 1640.0 & 1728.7 \\
\hline
\end{tabular}

In the composites with SWCNTs, $c_{12}$ and $c_{13}$ decrease for both low and high CNT concentrations, which depend on the CNT diameters and the CNT volume fractions. A larger decrease is seen for composites with larger CNT diameters. For example, in the case of $\mathrm{Ni} / \mathrm{SWCNT}(15,0)$ with high CNT volume fraction, the value for $c_{12}$ and $c_{13}$ is found to be 18 $\mathrm{GPa}$ less than that of $\mathrm{Ni}$. The $c_{23}$ on the other hand, remain similar to that of $\mathrm{Ni}$, with a few exceptions, where a minute increase is seen. For the composites with the MWCNT, $c_{12}$ and $c_{13}$ decrease for both low and high CNT volume fractions. For $c_{23}$, however, a small increase is seen for both volume fractions. The values of $c_{44}, c_{55}$, and $c_{66}$ decrease in the composites compared to pure $\mathrm{Ni}(125 \mathrm{GPa})$ and depend on the CNT concentration in the composites. A similar trend is seen for $c_{55}$ and $c_{66}$, although these show less variation than $c_{44}$. The Ni/MWCNT composites also show similar CNT concentration dependence for these elastic parameters, however, the decrease in $c_{44}$ is less in a relative sense. Note that $c_{44}, c_{55}$, and $c_{66}$ are equivalent to the shear modulus $(G)$.

To study the angular anisotropy of the elastic constants in $\mathrm{Ni} / \mathrm{CNT}$, we performed calculations in which the CNT is rotated about its tubular axis. Elastic constants from these calculations are similar to the original unrotated systems. While lack of anisotropy merits further investigation, the occurrence of a regular interfacial structural pattern seems unlikely because this interface forms between the large peripheral surface of the CNT that runs through the entire $7.4 \mathrm{~nm}$ length of the unit cell and the irregular curvature of the $\mathrm{Ni}$ matrix in the perpendicular directions.

The Young's modulus $(E)$ along the normal direction of the lattice plane is defined as the ratio of the stress to the strain along that direction. $E_{11}\left(E_{001}\right), E_{22}\left(E_{100}\right)$, and $E_{33}$ $\left(E_{010}\right)$ were calculated for cubic and orthorhombic lattices along the principal $(a, b$, and $c)$ axes. Poisson's ratio $\nu(n, m)$ of an elastic solid for any two specified general orthogonal unit vectors $n$ and $m$ is defined as $-\varepsilon(m) / \varepsilon(n)$, where $\varepsilon(m)$ is the transverse strain in the direction $m$ and $\varepsilon(n)$ is the axial strain in the direction $n$ due to a uniaxial tension of the material along the direction $n$. With the exception of isotropic elastic materials, Poisson's ratio for an anisotropic elastic material depends on the choice of $n$ and $m$ and was calculated from the elastic compliance values.

Figure 3 presents the calculated values of $E_{11}, E_{22}$, and $E_{33}$ for $\mathrm{Ni}$ and $\mathrm{Ni} / \mathrm{CNT}$ composites for two different CNT volume fractions. For pure $\mathrm{Ni}$, we calculated $E$ of $136.9 \mathrm{GPa}$ along the major directions. This agrees reasonably well with an experimental value of $120 \mathrm{GPa} .{ }^{49}$ For composites, in the

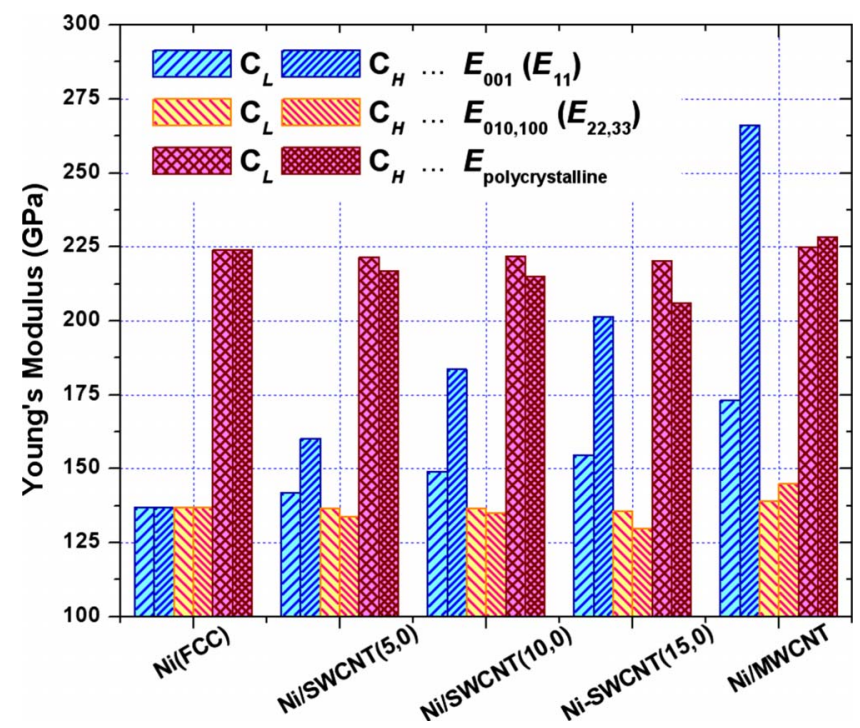

FIG. 3. (Color online) Calculated Young's modulus (GPa) for fcc $\mathrm{Ni}$ and $\mathrm{Ni} / \mathrm{CNT}$ composites with two different CNT fractions. The $E_{001}\left(E_{11}\right)$ is the Young's modulus for uniaxial loading parallel to CNT axis, the $E_{010}\left(E_{22}\right)$ or $E_{100}\left(E_{33}\right)$ are the Young's moduli in the directions transverse to the CNT axis in single-crystal systems, and $E_{\text {polycrystalline }}$ is the Young's modulus for polycrystalline aggregates. Composites with low CNT fractions are designated as $C_{\mathrm{L}}$ and composites with high $\mathrm{CNT}$ fractions are designated as $C_{\mathrm{L}}$. 


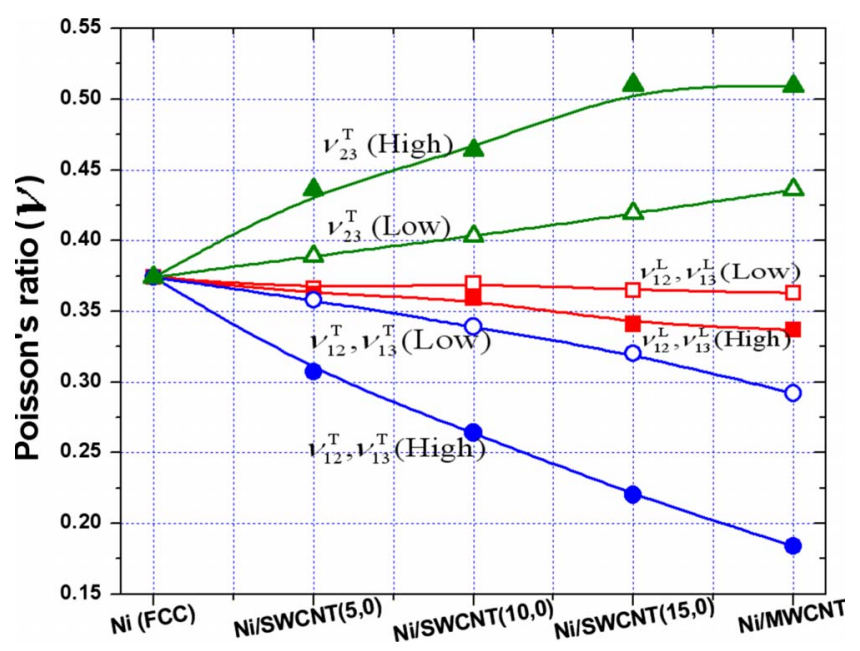

FIG. 4. (Color online) Calculated Poisson's ratio $(\nu)$ for fcc Ni and $\mathrm{Ni} / \mathrm{CNT}$ composites with low and high CNT volume fractions.

longitudinal direction (the direction of CNT alignments), $E_{11}$ $\left(E_{11}^{\mathrm{L}}\right)$ show a dramatic increase and follows the trend

$$
\mathrm{Ni} / \operatorname{SWCNT}(5,0)<\mathrm{Ni} / \mathrm{SWCNT}(10,0)
$$

$$
<\mathrm{Ni} / \mathrm{SWCNT}(15,0)<\mathrm{Ni} / \mathrm{MWCNT} \text {. }
$$

These increases depend on the CNT volume fractions of the composites and approximately proportional to the CNT volume fractions. Similar trend is seen for $E_{22}\left(E_{22}^{\mathrm{T}}\right)$ and $E_{33}$ $\left(E_{33}^{\mathrm{T}}\right)$. For SWCNTs, a slight but gradual decrease is seen for the $E$ values depending on the CNT diameter and volume fractions. Interestingly, $E_{22}^{\mathrm{T}}$ and $E_{33}^{\mathrm{T}}$ increases for $\mathrm{Ni} /$ MWCNT. For example, in this case, $E_{22}^{\mathrm{T}}$ (or $\left.E_{33}^{\mathrm{T}}\right)$ is increased by $2 \mathrm{GPa}$ for low CNT volume fraction and an average of 8 $\mathrm{GPa}$ for high CNT volume fraction, respectively, which suggests that Ni/CNT composites with significantly larger MWCNT diameter and with high CNT volume fractions might increase the Young's modulus in both longitudinal and transverse directions. However, verification of this hypothesis is beyond the scope of the present paper.

Figure 4 shows the calculated Poisson's ratio, $\nu$, for $\mathrm{Ni}$ and the Ni/CNT composites. Our predicted value for pure fcc $\mathrm{Ni}$ is 0.374 . Several unique Poisson's ratios can be defined for the composites depending on the orthogonal directions of the unit vector along the longitudinal direction and the transverse directions of the composites: (1) $\nu_{12}^{\mathrm{L}}$ and $\nu_{13}^{\mathrm{L}}$ involve the strains between the longitudinal direction and the transverse directions. Here, a uniaxial strain is applied in the longitudinal direction while the transverse directions respond to this freely. Usually this indicates contraction in the transverse direction during longitudinal elongation; (2) $\nu_{12}^{\mathrm{T}}$ and $\nu_{13}^{\mathrm{T}}$ are similar to $\nu_{12}^{\mathrm{L}}$ and $\nu_{13}^{\mathrm{L}}$, however, in this case a uniaxial strain is applied in the transverse direction while response (contraction) is expected in the longitudinal direction; and, (3) in $\nu_{23}^{T}$ both transverse directions are involved, however, a uniaxial strain is applied in one transverse direction and contraction is seen in the other transverse direction. Note, since similar values are found for $\nu_{12}^{\mathrm{L}}$ and $\nu_{13}^{\mathrm{L}}$, as well as $\nu_{12}^{\mathrm{T}}$ and $\nu_{13}^{\mathrm{T}}$, we show an average values for these.
From Fig. 4, the following trends are seen: (1) $\nu_{12}^{\mathrm{L}}$ or $\nu_{13}^{\mathrm{L}}$ show slight variation across the different Ni/CNTs systems starting from Ni/SWCNT(5,0) in the left to $\mathrm{Ni} /$ SWCNT $(15,0)$ and Ni/MWCNT on the right. In the composites with high CNT volume fraction, slight decrease is seen from left to right depending on CNT diameters. The largest decrease is seen for Ni/MWCNT. In this case, for high CNT volume fractions, $\nu_{12}^{\mathrm{L}}$ or $\nu_{13}^{\mathrm{L}}$ are found to be 0.34 , a value only marginally smaller than that of 0.37 of $\mathrm{Ni}$. (2) In $\nu_{12}^{\mathrm{T}}$ or $\nu_{13}^{\mathrm{T}}$, a significant and rapid decrease is seen across for the Ni/CNT systems studied (left to right in Fig. 4). Composites with high volume fraction show a far larger decrease. The Ni/MWCNT nanocomposites show larger decrease than any of the Ni/SWCNT composites. For example, for Ni/CNTs with a high CNT volume fraction, the $\nu_{12}^{\mathrm{T}}$ (or $\nu_{13}^{\mathrm{T}}$ ) is found to be $0.31,0.26,0.22$, and 0.18 , for $\mathrm{Ni} / \operatorname{SWCNT}(5,0)$, Ni/ SWCNT(10,0), Ni/SWCNT(15,0), and Ni/MWCNT, respectively. An interesting difference in trend is seen between the longitudinal $\nu_{12}^{\mathrm{L}}\left(\right.$ or $\left.\nu_{13}^{\mathrm{L}}\right)$ and the transverse $\nu_{12}^{\mathrm{T}}\left(\right.$ or $\left.\nu_{13}^{\mathrm{T}}\right)$. Here, CNT volume fractions dependency is seen for both of these, a much steeper decrease is seen for $\nu_{12}^{\mathrm{T}}$ (or $\nu_{13}^{\mathrm{T}}$ ) across the Ni/CNTs. (3) For $\nu_{23}^{T}$, a large but gradual increase is seen across the Ni/CNTs, which depend on the CNT diameters. This increase is also found to be CNT volume fraction dependent, with a larger increase for composites with high volume fractions. The variations in $\nu_{23}^{\mathrm{T}}$ indicate how Ni/CNTs with different kind of CNTs would respond when an uniaxial strain is applied along one transverse direction while other is being freely contracted. The significantly larger changes in $\nu_{12}^{\mathrm{T}}\left(\right.$ or $\left.\nu_{13}^{\mathrm{T}}\right)$ and $\nu_{23}^{\mathrm{T}}$ indicate that these composites have markedly different behavior along longitudinal and transverse directions when uniaxial strain is applied.

For all composites, the calculated shear modulus $(G)$ for single crystals decrease compared to nickel. For composites with high CNT volume fractions, a larger decrease is seen as compared to those with low volume fractions. For Ni/ SWCNT, both $G_{23}$ and $G_{13}$ or $G_{12}$ decrease with increasing CNT diameters.

\section{Polycrystalline elastic moduli}

Real materials are usually polycrystalline aggregates of randomly oriented single-crystal grains, each exhibiting single-crystalline elastic properties. These materials exhibit isotropic properties. The elastic moduli of polycrystalline $\mathrm{Ni} /$ CNT aggregates were computed by averaging appropriate single-crystal constants $\left(c_{i j}\right)$ using the expressions of Voigt ${ }^{50}$ and Reuss. ${ }^{51}$ These expressions provide upper and lower bounds for the averaged bulk and shear modulus. As shown thermodynamically by Hill, ${ }^{52}$ the average of the Voigt and Reuss bounds provide better estimates of the elastic moduli of polycrystalline materials. By treating the polycrystalline materials as aggregates of single crystals with random orientations, the isotropic polycrystalline elastic moduli can be computed as averages of anisotropic single-crystal elastic constants. The theoretical lower and upper bounds to the true polycrystalline bulk modulus $B$ and shear modulus $G$ are given by Reuss (assuming uniform stress throughout a polycrystal) and Voigt (assuming uniform strain) as follows: 
TABLE IV. Polycrystalline bulk (B) and shear (G) moduli calculated (in GPa) for Ni and nickel/CNT composites. The anisotropies of the bulk modulus along the $a$ axis with respect to the $b$ and $c$ axes are also given. The values are shown for Voigt (V), Reuss (R), and Hill (H) approximations. Low and High are the composites low and high CNT volume fractions.

\begin{tabular}{|c|c|c|c|c|c|c|c|c|c|c|c|c|}
\hline \multirow[t]{2}{*}{$\mathrm{Ni}(\mathrm{fcc})$} & \multicolumn{2}{|c|}{$G_{V}(95.2)$} & \multicolumn{2}{|c|}{$B_{V}(180.7)$} & \multicolumn{2}{|c|}{$G_{R}(78.04)$} & \multicolumn{2}{|c|}{$B_{R}(180.7)$} & \multicolumn{2}{|c|}{$G_{H}(86.6)$} & \multicolumn{2}{|c|}{$B_{H}(180.7)$} \\
\hline & Low & High & Low & High & Low & High & Low & High & Low & High & Low & High \\
\hline Ni/SWCNT $(5,0)$ & 93.3 & 89.6 & 180.9 & 181.4 & 77.6 & 77.0 & 181.1 & 181.2 & 85.5 & 83.3 & 181.1 & 181.3 \\
\hline Ni/SWCNT(10,0) & 93.0 & 87.7 & 180.6 & 180.4 & 78.3 & 77.5 & 180.5 & 179.7 & 85.6 & 82.6 & 180.5 & 180.0 \\
\hline Ni/SWCNT $(15,0)$ & 92.0 & 83.7 & 179.1 & 174.4 & 78.1 & 74.4 & 179.0 & 173.7 & 85.0 & 79.1 & 179.0 & 174.1 \\
\hline Ni/MWCNT & 93.2 & 92.6 & 182.2 & 186.7 & 80.7 & 83.8 & 182.0 & 183.2 & 86.9 & 88.2 & 182.0 & 184.9 \\
\hline
\end{tabular}

$$
\begin{aligned}
B_{R}=1 /\left[s_{11}+s_{33}+s_{33}+2\left(s_{12}+s_{23}+s_{13}\right)\right], \\
B_{V}=1 / 9\left(c_{11}+c_{33}+c_{33}\right)+2 / 9\left(c_{12}+c_{23}+c_{13}\right), \\
G_{R}=15 /\left[4\left(s_{11}+s_{33}+s_{33}\right)-4\left(s_{12}+s_{23}+s_{13}\right)\right. \\
\left.\quad+3\left(s_{44}+s_{55}+s_{66}\right)\right],
\end{aligned}
$$

$G_{V}=\left(c_{11}+c_{22}+c_{33}-c_{12}-c_{23}-c_{13}\right) / 15+\left(c_{44}+c_{55}+c_{66}\right) / 5$,

where $s_{i j}$ are elastic compliances and their values can be obtained through an inversion of the elastic constant matrix, $S=C^{-1}$. We estimated the true polycrystalline values by using the Voigt and Reuss, and Hill (VRH) approximations: ${ }^{52} B_{H}$ $=\left(B_{R}+B_{V}\right) / 2$ and $G_{H}=\left(G_{R}+G_{V}\right) / 2$. The average Young's modulus and Poisson's ratio for polycrystalline aggregates can then be calculated as $E_{\text {polycrystalline }}=\left(9 B_{H} G_{H}\right) /\left(3 B_{H}\right.$ $\left.+G_{H}\right)$ and $\nu_{\text {polycrystalline }}=\left(3 B_{H}-2 G_{H}\right) /\left(6 B_{H}+2 G_{H}\right)$.

Table IV shows the calculated bulk $\left(B_{H}\right)$ and shear $\left(G_{H}\right)$ moduli for the polycrystalline aggregates of $\mathrm{Ni}$ and the $\mathrm{Ni}$ / CNT composites using VRH approximations. We discuss the results obtained using the Hill average only. The calculated $B_{H}$ for polycrystalline nickel is identical to that of the corresponding single crystal and also agrees well with experiment. The bulk modulus of $\mathrm{Ni}$ determined from the BirchMurnaghan equation of state is also found to be identical. For Ni/CNT composites, no significant changes are seen in $B_{H}$ except for $\mathrm{Ni} / \mathrm{CNT}$, which show a slight increase of 1 $\mathrm{GPa}$ and $4 \mathrm{GPa}$, respectively, for low and high CNT volume fractions. The shear modulus $G_{H}$, which measures the ability of a material to resist transverse deformations, decreases slightly for Ni/SWCNTs as compared to pure Ni. For fcc nickel, the calculated $G_{H}$ is found to be $87 \mathrm{GPa}$, which agrees well with experiment. ${ }^{49}$ For Ni/MWCNT, these values increase slightly and depend on CNT volume fractions.

Pugh $^{53}$ introduced the ratio between the bulk and shear modulus, $B_{H} / G_{H}$, for polycrystalline phases as a measure of fracture/toughness in metals by considering that the shear modulus $G_{H}$ represents the resistance to plastic deformation while the bulk modulus $B_{H}$ represents the resistance to fracture. A high $B_{H} / G_{H}$ value is associated with ductility while a low value is associated with brittleness. The critical value that separates ductile and brittle materials is about 1.75. Our calculated $B_{H} / G_{H}$ for pure nickel is 2.1. For Ni/CNT composites, similar $B_{H} / G_{H}$ values are found. By considering the bulk modulus as a measure of the average bond strength and the shear modulus as a measure of the resistance to a change in bond angle by an external force, Tanaka et al. ${ }^{54}$ proposed that $G_{H} / B_{H}$ represents the relative directionality of the bonding in the material. It can be seen that there is a slight decrease in these ratios for Ni/SWCNTs. Composites with high CNT volume fractions show a greater decrease. For Ni/ MWCNT, there is no observable change with pure Ni. The $B_{H} / G_{H}$ and the $G_{H} / B_{H}$ values indicate that CNT do not significantly alter the above-mentioned properties as compared to fcc Ni.

The average Young's modulus for polycrystalline aggregates $\left(E_{\text {polycrystalline }}\right)$ calculated from the Hill's formula for pure $\mathrm{Ni}$ and the Ni/CNTs are presented graphically in Fig. 3. The Young's modulus in longitudinal $E_{001}\left(E_{11}\right)$ and transverse directions, $E_{010}, E_{100}\left(E_{22}, E_{33}\right)$ determined for singlecrystal specimens are also compared. For, Ni the value calculated using Hill's average is $222 \mathrm{GPa}$. This value is in excellent agreement with the experimental value of 213.1 $\pm 21.2 \mathrm{GPa}$, obtained for coarse-grained polycrystalline aggregates. ${ }^{49}$ In Ni/SWCNT composites, $E_{\text {polycrystalline }}$

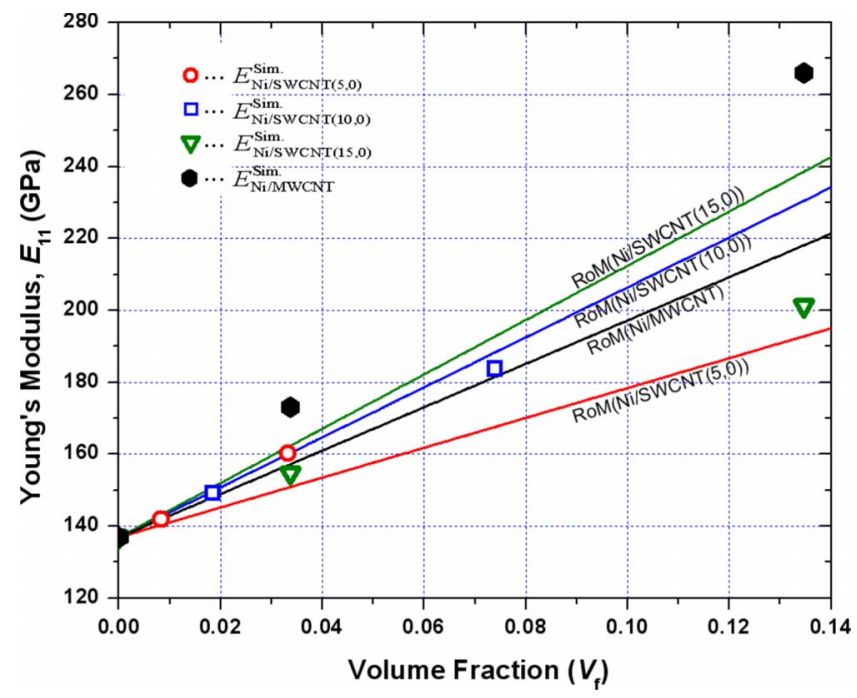

FIG. 5. (Color online) Young's modulus in longitudinal direction, $E_{11}$, (in $\mathrm{GPa}$ ) simulated and calculated using the rule-ofmixture (RoM) for Ni/SWCNT and Ni/MWCNT composites with low- and high CNT volume fractions. The straight lines for each CNT type are obtained by fitting the RoM data at low and high CNT volume fractions. The simulated values for each composite are presented with symbols. 
TABLE V. The isotropic bulk modulus ( $B$ in GPa) and directional bulk modulus along the orthorhombic crystallographic axes $a, b$, and $c\left(B_{a}, B_{b}\right.$, and $\left.B_{c}\right)$ for pure Nickel (fcc) and nickel/CNT composites. Low and High are the composites low and high CNT volume fractions.

\begin{tabular}{|c|c|c|c|c|c|c|c|c|}
\hline \multirow[t]{2}{*}{$\mathrm{Ni}(\mathrm{fcc})$} & \multicolumn{2}{|c|}{$B(180.7)$} & \multicolumn{2}{|c|}{$B_{a}(542.1)$} & \multicolumn{2}{|c|}{$B_{b}(542.1)$} & \multicolumn{2}{|c|}{$B_{c}(542.1)$} \\
\hline & Low & High & Low & High & Low & High & Low & High \\
\hline $\mathrm{Ni} / \mathrm{SWCNT}(5,0)$ & 181.1 & 181.2 & 560.8 & 599.8 & 530.9 & 516.3 & 538.8 & 522.5 \\
\hline Ni/SWCNT $(10,0)$ & 180.5 & 179.2 & 573.3 & 650.5 & 527.4 & 498.3 & 526.6 & 495.1 \\
\hline $\mathrm{Ni} / \mathrm{SWCNT}(15,0)$ & 179.0 & 173.7 & 572.6 & 629.5 & 520.4 & 479.7 & 521.2 & 479.9 \\
\hline Ni/MWCNT & 182.0 & 183.2 & 629.3 & 817.4 & 508.6 & 462.2 & 514.5 & 482.9 \\
\hline
\end{tabular}

decreases with increasing CNT diameter. Composites with higher CNT volume fractions show larger decreases. Ni/ MWCNT does not show an appreciable change in $E_{\text {polycrystalline }}$ for the composite with low volume fraction and a slight increase for composites with high volume fractions of CNT. Generally, $E_{\text {polycrystalline }}$ is much larger in magnitudes than the single crystal $E$. From these trends, we can conclude that embedding SWCNTs in the Ni matrix will decrease the polycrystalline Young's modulus $E_{\text {polycrystalline }}$ On the contrary, the polycrystalline Young's modulus might increase when MWCNTs are embedded in the Ni matrix.

Our calculated Poisson's ratio for polycrystalline nickel is 0.293 . This is in reasonable agreement with the experimental value of $0.31 .^{55}$ Surprisingly, we find similar values for $\mathrm{Ni} /$ CNT composites. For example, for $\mathrm{Ni} / \mathrm{SWCNT}(5,0), \mathrm{Ni} /$ SWCNT(10,0), Ni/SWCNT(15,0), and Ni/MWCNT composites with higher CNT fractions, the calculated Poisson's ratios are $0.301,0.301,0.303$, and 0.317 , respectively. They are $0.296,0.295,0.295$, and 0.294 for the same composites with lower CNT volume fractions. The calculated Poisson's ratios indicate that the $\mathrm{Ni} / \mathrm{CNT}$ composites remain similarly stable against shear as compared to pure $\mathrm{Ni}$.

\section{Elastic anisotropy}

Elasticity describes the response of a crystal under external strain and provides key information about the bonding characteristic between adjacent atomic planes and the anisotropic character of the solid. ${ }^{26}$ The shear anisotropic factors measure the degree of anisotropy in the bonding between atoms in different planes. Anisotropy in orthorhombic materials arises from the anisotropy in their shear and linear bulk moduli.

The shear anisotropic factor is defined as

$$
A_{1}=4 c_{44} /\left(c_{11}+c_{33}-2 c_{13}\right)
$$

for the $\{100\}$ shear planes in $\langle 011\rangle$ and $\langle 010\rangle$ directions,

$$
A_{2}=4 c_{55} /\left(c_{22}+c_{33}-2 c_{23}\right)
$$

for the $\{010\}$ shear planes in $\langle 101\rangle$ and $\langle 001\rangle$ directions, and

$$
A_{3}=4 c_{66} /\left(c_{11}+c_{22}-2 c_{12}\right)
$$

for the $\{001\}$ shear planes in $\langle 110\rangle$ and $\langle 010\rangle$ directions. For an isotropic crystal, the factors $A_{1}, A_{2}$, and $A_{3}$ must be one. The deviation from one is a measure of the degree of the elastic anisotropy of crystals.
The anisotropy of the bulk modulus along the $a$ axis with respect to $b$ and $c$ axes can be estimated using the following equations:

$$
A_{B_{b}}=\frac{B_{a}}{B_{b}}, \quad A_{B_{c}}=\frac{B_{a}}{B_{c}} .
$$

Note that a value of unity for these two fractions indicates elastic isotropy. In the above equations, $B_{a}, B_{b}$, and $B_{c}$ are the bulk moduli along different crystal axes, are defined as

$$
B_{a}=a \frac{d P}{d a}, \quad B_{b}=b \frac{d P}{d b}, \quad \text { and } \quad B_{c}=c \frac{d P}{d c}
$$

A practical measure of elastic anisotropy for polycrystalline materials is defined as follows:

$$
A_{B}=\frac{\left(B_{V}-B_{R}\right)}{\left(B_{V}+B_{R}\right)} \quad \text { and } \quad A_{G}=\frac{\left(G_{V}-G_{R}\right)}{\left(G_{V}+G_{R}\right)}
$$

where $B$ and $G$ are the bulk and shear moduli and the subscripts $V$ and $R$ denote the Voigt and Reuss limits. For these expressions, a value of zero identifies elastic isotropy and a value of $1(100 \%)$ is the largest possible anisotropy.

The bulk modulus and the directional bulk modulus are given in Table V. Note that the CNTs are aligned along the $a$ axis. It is clear that $B_{a}$ is higher than $B_{b}$ and $B_{c}$, which are nearly identical. In the composites, $B_{a}$ increases with volume fraction of CNT while $B_{b}$ and $B_{c}$ both decrease as compared to $\mathrm{Ni}$. The directional bulk modulus of $\mathrm{Ni}$ is $542 \mathrm{GPa}$ along all three axes. For Ni/MWCNT composites, the highest (817 $\mathrm{GPa}$ ) and lowest $(462 \mathrm{GPa})$ values for $B_{a}$ and $B_{b}$ or $B_{c}$ are seen for high CNT volume fractions. For Ni/SWCNTs, dependencies on the diameters are also seen.

The shear anisotropic factors are given in Table VI. For fcc Ni, we calculated a shear anisotropic factor $A_{s}$ is 2.52 while the value reported in the literature ${ }^{56}$ value is 2.63 . This indicates significant deviation from isotropy. We also see that the shear anisotropic factors $A_{1}, A_{2}$, and $A_{3}$ for the composites depend on CNT concentrations. Similar changes are seen for $A_{1}$ and $A_{3}$ where these values are found to be lower than pure nickel. A steeper decrease for these is seen for composite with high CNT volume fractions. For SWCNTs, a larger decrease is seen for CNTs with larger diameter. $A_{2}$ for the composites remains similar to that for pure nickel. 
TABLE VI. The shear anisotropic factors $A_{1}, A_{2}, A_{3}$, and $A_{G}$ (in $\%$ ), $A_{B}$ (in \%) for pure Nickel (fcc) and nickel/CNT composites with low and high CNT volume fractions.

\begin{tabular}{|c|c|c|c|c|c|c|c|c|c|c|c|c|c|c|}
\hline \multirow[t]{2}{*}{$\mathrm{Ni}(\mathrm{fcc})$} & \multicolumn{2}{|c|}{$A_{1}(2.516)$} & \multicolumn{2}{|c|}{$A_{2}(2.516)$} & \multicolumn{2}{|c|}{$A_{3}(2.516)$} & \multicolumn{2}{|c|}{$A_{B}(0.00)$} & \multicolumn{2}{|c|}{$A_{G}(9.875)$} & \multicolumn{2}{|c|}{$A_{B_{b}}(1)$} & \multicolumn{2}{|c|}{$A_{B_{c}}(1)$} \\
\hline & Low & High & Low & High & Low & High & Low & High & Low & High & Low & High & Low & High \\
\hline $\mathrm{Ni} / \mathrm{SWCNT}(5,0)$ & 2.452 & 2.155 & 2.485 & 2.409 & 2.370 & 2.026 & 0.005 & 0.047 & 9.237 & 7.542 & 1.056 & 1.162 & 1.040 & 1.148 \\
\hline Ni/SWCNT $(10,0)$ & 2.247 & 1.630 & 2.504 & 2.452 & 2.309 & 1.838 & 0.015 & 0.180 & 8.602 & 6.158 & 1.087 & 1.305 & 1.088 & 1.314 \\
\hline Ni/SWCNT $(15,0)$ & 2.113 & 1.269 & 2.525 & 2.539 & 2.228 & 1.657 & 0.020 & 0.209 & 8.145 & 5.874 & 1.101 & 1.315 & 1.098 & 1.311 \\
\hline Ni/MWCNT & 2.002 & 1.304 & 2.444 & 2.181 & 2.000 & 1.254 & 0.102 & 0.929 & 7.218 & 4.985 & 1.237 & 1.768 & 1.223 & 1.692 \\
\hline
\end{tabular}

From the anisotropy of the directional linear bulk modulus it is clear that fcc nickel does not show any directionality. However, due to the alignment of the CNTs, the nanocomposites show differences in the bulk moduli along different axes. For example, along the direction of CNT alignment (i.e., $a$ axis), there is an increase in the bulk modulus whereas in the transverse directions (i.e., $b$ and $c$ axes) decreases are seen compared to $\mathrm{Ni}$. These changes are more prominent at higher CNT concentrations. These results imply that the composites are less compressible along the direction of the CNT axis and more compressible along the transverse directions. The anisotropy of the bulk modulus $A_{B_{b}}$ and $A_{B_{C}}$ along the $a$ axis with respect to $b$ and $c$ axes shows that anisotropy increase with the increasing CNT diameter and CNT volume fraction. For $\mathrm{Ni}$, the bulk anisotropy $\left(A_{B}\right)$ is zero indicating no anisotropic compressibility. For composites, $A_{B}$ increases with increase in the CNT volume fraction and CNT diameters indicating an increase in compressibility. The maximum value for $A_{B}$ is seen for the Ni/MWCNT composite.

$A_{G}$ gives the relative magnitude of the actual elastic anisotropy possessed by a crystal. ${ }^{56}$ In the case of shear anisotropy $\left(A_{G}\right)$, MEAM Ni shows the maximum value of 9.88 while a value of 9.76 is reported in the literature. ${ }^{56}$ This suggests that $\mathrm{Ni}$ has large shear anisotropy. A decrease is seen for composites with larger CNT radii and volume fractions. Thus, it can be concluded that the composites are also elastically anisotropic. However, there are significant effects of CNTs within the composites, the $A_{G}$ decrease and larger decreases are seen for composites with higher CNT volume fractions.

It is interesting to note that the composites have the highest directional bulk modulus along the direction in which the nanotubes are aligned. In the transverse directions, the values are comparable. This indicates that the compressibility is smallest along the longitudinal direction and largest along the transverse direction. Along the CNT alignment direction, the bulk modulus increases compared to pure nickel and it decreases in the transverse directions. CNT concentration also affects the bulk modulus significantly.

\section{Interfacial characteristics}

We have closely monitored the motion of nickel and carbon atoms in Ni/SWCNT(10,0) with low CNT volume fraction at $0.0 \%$ and $0.2 \%$ applied strains. We find both nickel and carbon atoms move in concert with each other in the direction of applied strain. There is little or no movement of atoms in the transverse directions, except for a few atoms in the central portion of the cell where $\mathrm{Ni}$ atoms are found to perturb laterally in the perpendicular directions. In the vicinity of the nanotube, it is seen that first few Ni atoms move away and others shift toward the center during minimization in this region. The overall relative shifts of atomic positions during the loading can be quantitatively estimated using the root mean-square deviations (RMSDs) of the atoms in the simulation cell from those two strain limits. It is seen that the RMSD for Ni/CNT system is $0.0214 \AA$ in the longitudinal direction, which interestingly, is virtually identical to the RMSDs calculated for CNT and Ni atoms separately. Note that these RMSD calculations were performed by taking on the central portion of the original cells but reducing it by $\frac{1}{2}$ $\times \frac{1}{2} \times \frac{1}{2}$ in $x, y$, and $z$ directions. In the transverse directions, the RMSD is $0.0007 \AA$, indicating very small or no relative movement due to applied strain. These results suggest that both carbon and nickel atom move in concert during uniaxial loading in the longitudinal direction, without sliding of $\mathrm{C}$ atoms relative to the nickel atoms. This is possible in the case of a relatively strong Ni/CNT interface. This finding is also supported by our analysis of rule-of-mixture (RoM) results, discussed in the next section.

\section{Analysis of rule-of-mixture}

The properties of fiber-reinforced composites have been extensively studied to predict their elastic properties. ${ }^{57}$ One such approach is the RoM. ${ }^{58,59}$ For a CNT-reinforced nanocomposites, under uniaxial loading, the dependence of the Young's modulus on the CNT volume fraction can be theoretically estimated as

$$
E_{c}=V_{f} E_{f}+\left(1-V_{f}\right) E_{m},
$$

where $E_{c}$ is the predicted Young's modulus of the composite, $E_{f}$ is the Young's modulus of the fiber reinforcement (CNT), $E_{m}$ the Young's modulus of the matrix, and $V_{f}$ is the volume fraction of the CNTs.

We have estimated the Young's modulus in the longitudinal direction $\left(E_{11}\right)$ for all Ni/CNT composites and compared them with the results obtained directly from simulation. Figure 5 shows $E_{11}$ as a function of $V_{f}$ for Ni/CNT composites (here the full lines plots of the standard RoM expression). It is clear that the RoMs fit with the simulated results well and 
implicates a strong Ni/CNT interfacial interaction. The RoM $E_{11}$ increases linearly with volume fractions and also shows dependence with CNT's radii.

Comparison of the RoM estimated $E_{11}$ with simulated values in the case of SWCNTs shows an opposite trend. According to the RoM, we should expect $\operatorname{SWCNT}(15,0)$ to give the largest value of $E_{11}$ compared to that of SWCNT $(5,0)$. However, simulated results predict the opposite. $\operatorname{SWCNT}(10,0)$ shows a more remarkable agreement between calculated and simulated values than any other CNTs. Note that the plots in Fig. 5 are very useful as these can be used to predict the modulus for CNT with any diameter and volume fraction.

To reasonably compare the different CNT composites, one can use the rate of increase in Young's modulus with volume fraction $\left(d E_{11} / d V_{f}\right)$ as a convenient yardstick for assessing the effect of reinforcement (CNT effectiveness). This useful parameter shows the magnitude of the increase in stiffness and the CNT content required achieving that increase. For all four composites, the simulated $E_{11}$ increases approximately linearly with volume fraction of CNT. The $d E_{11} / d V_{f}$ are 707 $\mathrm{GPa}, 629 \mathrm{GPa}, 474 \mathrm{GPa}$, and $951 \mathrm{GPa}$ for Ni/SWCNT $(5,0)$, Ni/SWCNT(10,0), Ni/SWCNT(15,0), and Ni/MWCNT, respectively. The highest value of $d E_{11} / d V_{f}(951 \mathrm{GPa})$ was found with the MWCNT. Using the RoM data, the enhancement $d E_{11} / d V_{f}$ is found to be $415 \mathrm{GPa}, 695 \mathrm{GPa}, 755 \mathrm{GPa}$, and $603 \mathrm{GPa}$, respectively, for $\mathrm{Ni} / \mathrm{SWCNT}(5,0), \mathrm{Ni} /$ SWCNT(10,0), Ni/SWCNT(15,0), and Ni/MWCNT. This opposite trend between the RoM and simulated results suggests that the reinforcement mechanism is not only from mixing alone. This deviation likely arises from the large surface-area-to-volume ratios of CNTs that affect matrix properties beyond what would be predicted from RoM calculations.

It is known that the performance of a reinforced composite depends on the interfacial characteristics of the reinforcement fillers and the matrix materials. ${ }^{60}$ For example, strong adhesion forces at the interface lead to superior mechanical properties. It also critically depends on the effectiveness of the interfacial stress transfer, which in turn depends on the nature and strength of the interface. Chemical bonding, nanomechanical interlocking, and nonbonded (van der Waals and electrostatic) interactions yield strong interaction between a polymer matrix and CNTs. Furthermore, chemical functionalization of the filler surface and increasing nonbonded attractive interactions between the filler and the matrix increases such interfacial adhesion and improve the mechanical properties of composites. For most polymers, the polymer/CNT interfacial stress transfer is low with values typically less than $50 \mathrm{MPa} .{ }^{61}$

For metal/CNT interfaces, above-discussed information is not available. The large increase in $E_{11}$ for Ni/CNT composites indicates that there exists a surprisingly strong adhesive force between the CNT and the Ni matrix. An applied load is largely transferred to the reinforcing CNTs, indicating an effective stress transfer across the Ni-CNT interfaces that in turn enhance the mechanical response. We would like to emphasize that a thorough investigation using high-level theories is necessary to fully understand the chemistry at the Ni-CNT interfaces. Recent first-principles calculations on different structural models (e.g., top fcc, bridge top, etc.) of graphene on $\mathrm{Ni}(111)$ show very weak or no binding interaction between graphene and $\mathrm{Ni}$ surface, ${ }^{62,63}$ which may be due to shortcomings of current levels of density-functional theory utilized in solid-state calculations. Simulations using novel and adequate theory to rationalize the interfacial behavior in $\mathrm{Ni} / \mathrm{CNT}$ composites are beyond reach at this time due to the sheer size of these systems.

\section{CONCLUSIONS}

A MEAM formalism has been used to investigate the mechanical properties of Ni/CNT nanocomposites made by incorporating SWCNTs with three different diameters and a MWCNT into fcc Ni matrices. Two different volume fractions for each CNT were used to assess the CNT concentration effects upon mechanical properties of the composites. Using quasistatic energy-minimization methods and periodic boundary conditions, the single-crystal elastic constants, Young's and shear moduli, Poisson's ratios for pure nickel matrix, and Ni/CNT composites were calculated. The elastic anisotropy in the composites was also systematically investigated. The volume fraction dependence of the Young's modulus was analyzed using rule-of-mixture and compared that with the simulated values.

Calculations show that pristine SWCNTs have high Young's modulus values of $0.55 \mathrm{TPa}, 0.83 \mathrm{TPa}$, and $0.89 \mathrm{TPa}$ for the $\operatorname{SWCNT}(5,0), \operatorname{SWCNT}(10,0)$ and $\operatorname{SWCNT}(15,0)$, respectively. The MWCNT made from the above three SWCNTs show a Young's modulus of $0.74 \mathrm{TPa}$. These values are in reasonable agreement with the observed and theoretically predicted values published in the literature.

The results for Ni/CNT composites are primarily compared with the results for a pure fcc nickel matrix. Our calculations predict that all Ni/CNTs satisfy the conditions for mechanical stability. In the cases of single-crystal Ni/CNT composites, the Young's modulus shows a significant increase in the longitudinal direction (the direction of CNT alignment) and a moderate decrease in the transverse directions. It is found that CNT volume fractions have large effects on the single-crystal Young's modulus. For pure fcc nickel, our calculated Young's modulus is $137 \mathrm{GPa}$. In the composites with high CNT volume fraction, the singlecrystal Young's modulus along the longitudinal direction is found to be $160 \mathrm{GPa}, 183 \mathrm{GPa}$, and $201 \mathrm{GPa}$ for $\mathrm{Ni} /$ SWCNT(5,0), Ni/SWCNT(10,0), and Ni/SWNCT $(15,0)$, respectively. Ni/MWCNT shows the highest value of $266 \mathrm{GPa}$ for Young's modulus in same direction when high CNT volume fractions are used. In the transverse directions, the Young's modulus decreases by 1-7 GPa for Ni/SWCNTs depending on the CNT volume fraction and. For Ni/MWCNT however, 3-12 GPa increase is seen.

For the composites with SWCNTs, the polycrystalline Young's modulus decreases with increasing CNT diameters and CNT volume fractions. In the Ni/MWCNT nanocomposite, however, a slight increase is seen at high CNT volume fractions, indicating possibility of fabricating composites with enhanced overall mechanical properties when MWCNTs with sufficiently larger size and volume fractions were used. The polycrystalline shear modulus remain similar 
to pure nickel for the composites with Ni/SWCNTs, however, slight increase is seen for Ni/MWCNT composite with higher CNT volume fractions. The result indicates that $\mathrm{Ni}$ / CNT composites with embedded single-walled nanotubes with large CNT volume fraction would give slightly inferior mechanical properties than that of pure nickel. However, slightly enhanced mechanical properties may be expected when MWCNT with sufficiently large diameter and volume fractions are used.

Due to aligned CNTs, the composites show difference in the bulk modulus along different axes. The present calculations show that the bulk modulus along the longitudinal direction is much higher than the transverse directions and increases with the CNT volume fractions. The shear anisotropic factors $\left(A_{1}, A_{2}\right.$, and $\left.A_{3}\right)$ for the composites also depend on the CNT concentrations and are found to depend on the directions of CNT alignment. The bulk anisotropy $\left(A_{B}\right)$ increases with increase in the CNT volume fraction and CNT diameter, indicating an increase in compressibility. The maximum value for $A_{B}$ is seen for Ni/MWCNT nanocomposite. A decrease is seen in the shear anisotropy for composites with larger CNT radii and volume fractions.
The observations that (1) the Young's moduli obtained from the atomistic simulations and rule-of-mixture agree very well, (2) the reinforcement values, estimated from the rate of increase in the longitudinal Young's modulus as a function of CNT volume fraction, are remarkably high, and, (3) the $\mathrm{Ni}$ and $\mathrm{C}$ atoms at the $\mathrm{Ni} / \mathrm{CNT}$ interface move in concert, in the direction of applied strain, suggest a very strong $\mathrm{Ni} / \mathrm{CNT}$ interface in the $\mathrm{Ni} / \mathrm{CNT}$ composites.

\section{ACKNOWLEDGMENTS}

The authors gratefully acknowledge financial support from the Air Force Research Laboratory (Contract No. FA8650-08-C-5226, Manager Jay Tiley). The support from the National Science Foundation for computing infrastructure (Grants No. CHE-0342824 and No. CHE-0741936) and for studying deformation in light-weight materials (Grant No. CMMI-0846444), and the CASCaM for computational sources are gratefully acknowledged. We acknowledge support from UNT through the Material Modeling Research Cluster. We would also like to thank Kyeongjae Cho (UTDallas) for providing his MEAM parameters before their publication.
*Author to whom correspondence should be addressed; jamal.uddin@unt.edu

${ }^{1}$ S. Iijima, Nature (London) 354, 56 (1991).

${ }^{2}$ S. I. Cha, K. T. Kim, S. N. Arshad, C. B. Mo, and S. H. Hong, Adv. Mater. (Weinheim, Ger.) 17, 1377 (2005).

${ }^{3}$ T. Laha, S. Kuchibhatla, S. Seal, W. Li, and A. Agarwal, Acta Mater. 55, 1059 (2007).

${ }^{4}$ S. Yamanaka, A. Kawasaki, H. Sakamoto, Y. Mekuchi, M. Kuno, and T. Tsukada, J. Jpn. Inst. Met. 70, 630 (2006).

${ }^{5}$ S. R. Bakshi, J. E. Tercero, and A. Agarwal, Composites 38, 2493 (2007).

${ }^{6}$ Y. Han and J. Elliott, Comput. Mater. Sci. 39, 315 (2007).

${ }^{7}$ T. Kuzumaki, K. Miyazawa, H. Ichinose, and K. Ito, J. Mater. Res. 13, 2445 (1998).

${ }^{8}$ D. D. L. Chung, Carbon Fiber Composites (ButterworthHeinemann, Boston, 1994), Chap. 7.

${ }^{9}$ S. P. Rawal, JOM 53, 14 (2001).

${ }^{10}$ Y. Sun, J. Sun, M. Liu, and Q. Chen, Nanotechnology 18, 505704 (2007).

${ }^{11}$ S. J. V. Frankland, A. Caglar, D. W. Brenner, and M. Griebel, J. Phys. Chem. B 106, 3046 (2002).

${ }^{12}$ R. Zhu, E. Pan, and A. K. Roy, Mater. Sci. Eng., A 447, 51 (2007).

${ }^{13}$ A. Pineau and S. D. Antolovich, Eng. Failure Anal. 16, 2668 (2009).

${ }^{14}$ Superalloys II-High Temperature Materials Aerospace and Industrial Power, edited by C. T. Sims and W. C. Hagel (Wiley, New York, USA, 1987).

${ }^{15}$ M. S. Daw and M. I. Baskes, Phys. Rev. Lett. 50, 1285 (1983).

${ }^{16}$ M. S. Daw and M. I. Baskes, Phys. Rev. B 29, 6443 (1984).

${ }^{17}$ M. I. Baskes, Phys. Rev. B 46, 2727 (1992).

${ }^{18}$ J. H. Rose, J. R. Smith, F. Guinea, and J. Ferrante, Phys. Rev. B
29, 2963 (1984)

${ }^{19}$ B.-J. Lee, M. I. Baskes, H. Kim, and Y. K. Cho, Phys. Rev. B 64, 184102 (2001).

${ }^{20}$ B.-J. Lee and M. I. Baskes, Phys. Rev. B 62, 8564 (2000).

${ }^{21}$ B.-J. Lee, J.-H. Shim, and M. I. Baskes, Phys. Rev. B 68, $144112(2003)$.

${ }^{22}$ W. Xiao, M. I. Baskes, and K. J. Cho, Surf. Sci. 603, 1985 (2009).

${ }^{23}$ J. Cai, C. Y. Wang, T. Yu, and S. Yu, Phys. Scr. 79, 025702 (2009).

${ }^{24}$ J. P. Lu, Phys. Rev. Lett. 79, 1297 (1997).

${ }^{25}$ J. Cai, R. F. Bie, X. M. Tan, and C. Lu, Physica B 344, 99 (2004).

${ }^{26}$ P. Ravindran, L. Fast, P. A. Korzhavyi, B. Johansson, J. Wills, and O. Eriksson, J. Appl. Phys. 84, 4891 (1998).

${ }^{27}$ P. M. Agrawal, B. S. Sudalayandi, L. M. Raff, and R. Komanduri, Comput. Mater. Sci. 38, 271 (2006).

${ }^{28}$ M.-F. Yu, O. Lourie, M. J. Dyer, K. Moloni, T. F. Kelly, and R. S. Ruoff, Science 287, 637 (2000).

${ }^{29}$ B. I. Yakobson, C. J. Brabec, and J. Bernholc, Phys. Rev. Lett. 76, 2511 (1996).

${ }^{30}$ F. Li, H. M. Cheng, S. Bai, G. Su, and M. S. Dresselhaus, Appl. Phys. Lett. 77, 3161 (2000).

${ }^{31}$ B. G. Demczyk, Y. M. Wang, J. Cumings, M. Hetman, W. Han, A. Zettle, and R. O. Ritchie, Mater. Sci. Eng., A 334, 173 (2002).

${ }^{32}$ T. C. Chang and H. J. Gao, J. Mech. Phys. Solids 51, 1059 (2003).

${ }^{33}$ J.-P. Salvetat, G. A. D. Briggs, J.-M. Bonard, R. R. Bacsa, A. J. Kulik, T. Stöckli, N. A. Burnham, and L. Forró, Phys. Rev. Lett. 82, 944 (1999).

${ }^{34}$ Y. Jin and F. G. Yuan, Compos. Sci. Technol. 63, 1507 (2003). 
${ }^{35}$ T. W. Tombler, C. Zhou, L. Alexseyev, J. Kong, H. Dai, L. Liu, C. S. Jayanthi, M. Tang, and S. Y. Wu, Nature (London) 405, 769 (2000).

${ }^{36}$ E. Hernández, C. Goze, P. Bernier, and A. Rubio, Phys. Rev. Lett. 80, 4502 (1998).

${ }^{37}$ A. Krishnan, E. Dujardin, T. W. Ebbesen, P. N. Yianilos, and M. J. Treacy, Phys. Rev. B 58, 14013 (1998).

${ }^{38}$ Z. C. Tu and Z. C. Ou-yang, Phys. Rev. B 65, 233407 (2002).

${ }^{39}$ G. Zhou, W. H. Duan, and B. L. Gu, Chem. Phys. Lett. 333, 344 (2001).

${ }^{40}$ S. Ogata and Y. Shibutani, Phys. Rev. B 68, 165409 (2003).

${ }^{41}$ A. Pullen, G. L. Zhao, D. Bagayoko, and L. Yang, Phys. Rev. B 71, 205410 (2005).

${ }^{42}$ M. Rossi and M. Meo, Compos. Sci. Technol. 69, 1394 (2009).

${ }^{43}$ T. Narita and K. Shintani, Making Functional Materials with Nanotubes, MRS Symposia Proceedings No. 706 (Materials Research Society, Pittsburgh, 2002), p. Z971.

${ }^{44}$ O. Lourie and H. D. Wagner, J. Mater. Res. 13, 2418 (1998).

${ }^{45}$ C. A. Cooper and R. J. Young, Proc. SPIE 4098, 172 (2000).

${ }^{46}$ C. Y. Wang and L. C. Zhang, Nanotechnology 19, 075705 (2008).

${ }^{47}$ D. C. Wallace, Thermodynamics of Crystals (Wiley, New York, 1972).

${ }^{48}$ C. Kittel, Introduction to Solid State Physics (Wiley, New York, 1996).

${ }^{49}$ M. Kopycinska-Müller, A. Caron, S. Hirsekorn, U. Rabe, H. Natter, R. Hempelmann, R. Birringer, and W. Arnold, Z. Phys.
Chem. 222, 471 (2008).

${ }^{50}$ W. Voigt, Lehrbuch der Kristallphysik (Teubner, Leipzig, 1928), pp. 716-761.

${ }^{51}$ A. Reuss, Z. Angew. Math. Mech. 9, 49 (1929).

${ }^{52}$ R. Hill, Proc. Phys. Soc., London, Sect. A 65, 349 (1952).

${ }^{53}$ S. F. Pugh, Philos. Mag. 45, 823 (1954).

${ }^{54}$ K. Tanaka, K. Okamoto, H. Inui, Y. Minonishi, M. Yamaguchi, and M. Koiwa, Philos. Mag. A 73, 1475 (1996).

${ }^{55}$ W. D. Callister, Jr., Materials Science and Engineering: An Introduction (Wiley, USA, 2003).

${ }^{56}$ D. H. Chung and W. R. Buessem, J. Appl. Phys. 38, 2010 (1967).

${ }^{57}$ T. W. Chou, Microstructural Design of Fiber Composites (Cambridge University Press, Cambridge, 1992).

${ }^{58}$ M. O’Regan, D. F. Akay, and B. Meenan, Compos. Sci. Technol. 59, 419 (1999).

${ }^{59}$ S. W. Tsai, Composite Design, 4th ed. (Think Composites, Dayton, $\mathrm{OH}, 1988)$.

${ }^{60}$ F. H. Zhang, R. G. Wang, X. D. He, C. Wang, and L. N. Ren, J. Mater. Sci. 44, 3574 (2009).

${ }^{61}$ A. H. Barber, S. R. Cohen, and H. D. Wagner, Appl. Phys. Lett. 82, 4140 (2003).

${ }^{62}$ M. Fuentes-Cabrera, M. I. Baskes, A. V. Melechko, and M. L. Simpson, Phys. Rev. B 77, 035405 (2008).

${ }^{63}$ G. Bertoni, L. Calmels, A. Altibelli, and V. Serin, Phys. Rev. B 71, 075402 (2005). 